\title{
O Modelo de Coping de Folkman e Lazarus: Aspectos Históricos e Conceituais
}

\author{
Coping Model of Folkman and Lazarus: Historical and Conceptual Aspects \\ El Modelo de Coping de Folkman y Lazarus: Aspectos Históricos y Conceptuales
}

\author{
Ewerton Naves Dias ${ }^{1}$ \\ José Luís Pais-Ribeiro \\ Universidade do Porto, Portugal
}

\begin{abstract}
Resumo
Coping pode ser definido como um conjunto de estratégias cognitivas e comportamentais desenvolvidas pelas pessoas para lidar com as exigências internas e externas da relação entre o indivíduo e o ambiente. Essas estratégias estão relacionadas com a saúde mental, uma vez que podem moderar o impacto das adversidades ao longo da vida, além de aumentar os níveis de bemestar psicológico e reduzir o sofrimento. Este trabalho consiste em um estudo de revisão narrativa da literatura sobre o modelo de coping de Folkman e Lazarus. Concluiu-se que a teoria de coping proposta por Folkman e Lazarus continua a ser uma importante ferramenta para explicar as estratégias utilizadas pelas pessoas diante de um evento estressante, bem como para desvelar intervenções a fim de ajudar os indivíduos a lidarem melhor com a situação de estresse.
\end{abstract}

Palavras-chave: coping, estratégias de coping, estresse

\begin{abstract}
Coping can be defined as a set of cognitive and behavioral strategies developed by people to deal with internal and external requirements of the relationship between the individual and the environment. These strategies are related to the mental health, once they can moderate the impact of the adversities throughout life, besides increasing the levels of psychological well-being and reducing the suffering. This work consists in a study of narrative review of the literature about the coping model of Folkman and Lazarus. It was concluded that the coping theory proposed by Folkman and Lazarus is still an important tool to explain the strategies used by people in face of a stressful event, as well as to exhibit interventions in order to help individuals to deal better with the stressful situation.
\end{abstract}

Keywords: coping, coping strategies, stress

\section{Resumen}

Coping puede ser definido como un conjunto de estrategias cognitivas y conductuales desarrolladas por las personas para hacer frente a las exigencias internas y externas de la relación entre el individuo y el ambiente. Estas estrategias están relacionadas con la salud mental, ya que pueden moderar el impacto de las adversidades a lo largo de la vida, además de aumentar los niveles de bienestar psicológico y reducir el sufrimiento. Este trabajo consiste en un estudio de revisión narrativa de la literatura sobre el modelo de coping de Folkman y Lazarus. Se concluyó que la teoría de coping propuesta por Folkman y Lazarus sigue siendo una importante herramienta para explicar las estrategias utilizadas por las personas ante un evento estresante, así como para desvelar intervenciones para ayudar a los individuos a lidiar mejor con la situación de el estrés.

Palabras clave: coping, estrategias de coping, el estrés

\section{Introdução}

O presente estudo descreve aspectos teóricos e conceituais do modelo de coping proposto por Folkman e Lazarus. Numa perspectiva cognitiva e comportamental, os autores definem coping, sinteticamente, como as estratégias que as pessoas utilizam para se adaptar às circunstâncias adversas decorridas ao longo da vida. Tais estratégias possuem o potencial de impactar, de forma positiva ou negativa, a saúde física e mental das pessoas, com capacidade

\footnotetext{
${ }^{1}$ Endereço de contato: Universidade de Mogi das Cruzes - Av. Dr. Cândido Xavier de Almeida Souza, 200 - Centro Cívico, Mogi das Cruzes, SP, CEP 08780-911, Brasil. Contato: (11) 97313-1331 E-mail: ewertonnaves@gmail.com
} 
de modificar a evolução do estresse, seja evitando a situação estressora ou a confrontando-a (Lazarus \& Folkman, 1984). Estudos sobre esse fenômeno têm despertado o interesse de pesquisadores. Os resultados mais consistentes dessas pesquisas encontrados na literatura evidenciam o efeito e a correlação entre as estratégias de coping com o estresse e a saúde mental, demonstrando que o coping pode estar associado fortemente a resultados psicológicos positivos (Endler \& Parker, 1990; Folkman, Lazarus, Dunkel-Schetter, DeLongis \& Gruen, 1986; Lazarus \& Folkman, 1984; Seiffge-Krenke, 1993). Brantley e Jones (1993), Chaves, Cade, Montovani, Leite, \& Spire (2000) comentam que o efeito desse fenômeno pode interferir mais sobre o desenvolvimento da doença física e psicológica do que propriamente a presença ou não dos principais fatores de estresse (Brantley \& Jones, 1993, Chaves et al., 2000).

Holroyd e Lazarus (1982) complementam que a capacidade e a maneira como o indivíduo utiliza as estratégias de coping podem alterar o funcionamento biológico e, portanto, afetar os resultados de saúde através de uma variedade de mecanismos. Por exemplo, podem influenciar as respostas ao estresse neuroendócrino, contribuindo assim para mudanças na saúde, nos comportamentos de risco ou nas alterações a respostas cognitivas ou comportamentais da pessoa. Postula-se então que os fatores psicológicos, incluindo o estresse e o coping, são capazes de afetar a saúde do indivíduo, principalmente por meio de mecanismos fisiológicos ou de modificação de comportamentos. Em um estudo de revisão da literatura, Brantley e Garrett (1993) resumiram os modelos propostos de estresse e doença e concluíram que os comportamentos de alto risco podem causar mudanças no funcionamento fisiológico, diminuição na resistência a doenças, hipersensibilidade neurológica ou enfrentamento inadequado. Pesquisas examinando a relação específica entre o estresse e a doença apontam correlações entre o estresse psicológico e a apresentação de sintomas, tanto agudos quanto crônicos, com maior evidência nas doenças infecciosas, câncer, doenças cardiovasculares e crônicas, como diabetes, asma e perturbações gastrointestinais.

Nos últimos tempos, tem sido observado um crescimento considerável no número de estudos sobre o processo de coping. Entretanto, apesar desse ganho quantitativo, ainda existem lacunas na literatura sobre as questões teóricas, conceituais e práticas que envolvem o fenômeno. Com isso, os pesquisadores têm encontrado dificuldades para investigar e chegar a um consenso sobre o respectivo construto, o que de certa forma acaba por acentuar a existência de dilemas e debates sobre o processo de coping e suas implicações para a saúde, bem-estar e qualidade de vida das pessoas (Antoniazzi, Dell'aglio, \& Bandeira, 1998).

Diante das considerações apresentadas e, sobretudo, pelo potencial que as estratégias de coping possuem para intervir no processo saúde-doença, estudos como este, que pretendem abordar as questões históricas e conceituais do modelo de coping, são fundamentais e oportunos, pois podem ajudar a superar os dilemas conceituais que ainda envolvem esse constructo.

\section{Método}

Trata-se de uma revisão narrativa da literatura sobre aspectos históricos e conceituais do modelo de coping proposto por Folkman e Lazarus.

Os estudos de revisão narrativa são publicações bastante amplas, apropriadas para descrever o desenvolvimento ou o "estado da arte" sobre um determinado assunto, sob o ponto 
de vista teórico ou contextual. As revisões narrativas não informam as fontes de informação utilizadas, a metodologia para busca das referências nem os critérios utilizados na avaliação e seleção dos trabalhos (Bernardo, Nobre, \& Jatene, 2004). Basicamente, constituem-se na análise da literatura publicada em livros, artigos de revistas impressas e/ou eletrônicas, bases de dados, teses, entre outros. Essa categoria de estudo tem um papel fundamental, pois permite ao leitor adquirir e atualizar o conhecimento sobre uma temática específica (Rother, 2007).

\section{Aspectos Históricos sobre o Modelo de Coping}

A história do conceito de coping acompanha a psicologia desde os seus primórdios, focando na forma como as pessoas se adaptam às circunstâncias adversas da vida. O termo coping emerge da investigação da psicologia sobre o estresse, ao concluir que este não é simplesmente um processo automático de estímulo-resposta, mas sim influenciado por fatores mediadores que podem ser internos e externos. A partir desse momento, os estudos sobre esses fatores psicológicos do estresse (percepção da ameaça, avaliação, estratégias de enfrentamento e adaptação) levaram à formulação do coping como elemento integrante do processo de estresse (Pais-Ribeiro, 2005).

Após essa fase inicial, começaram a surgir na literatura novos estudos sobre essa temática, abordando o coping em contextos e práticas diversas e, com isso, dando origem a outras linhas de pensamento. Suls, David, e Harvey (1996) comentam que podemos encontrar na história do coping três gerações principais de pesquisadores com diferentes linhas de pensamento, tanto no que diz respeito aos aspectos teóricos quanto aos metodológicos.

A Primeira Geração é representada pelos pesquisadores psicanalistas vinculados à psicologia do ego, que conceberam o coping como o uso dos mecanismos de defesa, motivados interna e inconscientemente como forma de lidar com conflitos sexuais e agressivos (Vaillant, 1994). Os principais mecanismos de defesa propostos por S. Freud no século IX incluem a dissociação, a repressão e o isolamento (A. Freud, 1937; S. Freud, 1964), e para o autor, todos eram considerados patológicos (Parker \& Endler, 1992). Após essa perspectiva inicial, algumas distinções foram sendo feitas no sentido de diferenciar os mecanismos de defesa do coping propriamente dito. A principal modificação foi a distinção entre os comportamentos associados a esses mecanismos, classificados como rígidos, inadequados com relação à realidade externa, originários de questões do passado e derivados de elementos inconscientes. Já os comportamentos associados ao coping foram classificados como mais flexíveis e propositais, adequados à realidade e orientados para o futuro, com derivações conscientes (Haan, 1963, 1977). Nos dias de hoje, essa linha de pensamento não é mais dominante na literatura; entretanto ainda podemos encontrar adeptos a essa perspectiva do ego para o coping, sobretudo entre os pesquisadores interessados na psicopatologia da personalidade (Vaillant, 1977, 1994).

Já a Segunda Geração do coping teve início por volta dos anos de 1960, estendendo-se aproximadamente pelas próximas duas décadas. Essa nova geração foi caracterizada principalmente pelo trabalho de Richard Lazarus e seus associados. Os pesquisadores dessa linha de pensamento apontaram para uma nova perspectiva do modelo de coping e passaram a conceituar o fenômeno como um processo transacional entre a pessoa e o ambiente, dando 
ênfase no processo tanto quanto nos traços de personalidade, com destaque para o comportamento e seus determinantes cognitivos e situacionais (Lazarus, 1966; Folkman \& Lazarus, 1980, 1985). Para os autores dessa linha, o coping é centrado no processo, e não no traço, e distingue-se dos comportamentos automáticos adaptativos. Nesse sentido, usam o termo esforços para salientar o processo em vez do resultado e o termo gerir para evitar a ligação entre coping e sucesso, pois, em outras palavras, o esforço de coping pode não ser bem-sucedido e, então, não se pode defini-lo com base no sucesso do processo (Lazarus, DeLongis, Folkman, \& Gruen, 1985).

Lazarus (1993) explica que essa perspectiva se caracteriza por ser microanalítica, contextual e orientada para o processo e que possui nomeadamente quatro características: 1Descrição de pensamentos e ações que ocorrem numa dada situação estressante ou em vários estádios dessa situação; 2- Avaliação do coping não questiona o que a pessoa devia ou podia fazer ou pensar, ou o que costuma fazer numa dada situação, mas sim o que se passou num contexto particular, assumindo que o que aconteceu muda com o tempo ou com a alteração da situação ou contexto (é um processo). Isto ocorre como resultado de uma interação ativa entre a pessoa e o meio, com base numa relação psicológica; 3- Avaliação do coping implica que se avalie o mesmo indivíduo em vários contextos e momentos para identificar a estabilidade e a mudança nas ações e pensamentos relacionados ao coping; 4- Deve haver prudência na avaliação se um processo de coping é bom ou mau, pois isto dependente da pessoa, dos seus objetivos, dos resultados procurados e das suas preocupações.

A Segunda Geração é reconhecida como responsável por importantes avanços nessa área de conhecimento, gerando variadas publicações sobre o tema (Folkman \& Lazarus, 1980, 1985). A partir desse período, o coping passou a ser considerado fundamentalmente como um processo de resposta consciente ou reação a um acontecimento externo negativo (em oposição ao coping interno de Freud) (Parker \& Endler, 1992). Em resumo, os estudiosos da Primeira Geração acreditam que o enfrentamento seja uma característica da personalidade, representada principalmente pelo referencial teórico psicanalítico; enquanto isso, a Segunda Geração considera as estratégias de enfrentamento um processo dinâmico utilizado durante os eventos estressantes.

A Terceira Geração, por sua vez, teve início em meados da década de 1990. Os pesquisadores desse movimento focam no estudo das convergências entre coping e personalidade (Suls et al., 1996). Essa tendência tem sido motivada, em parte, pelo corpo cumulativo de evidências que indicam que fatores situacionais não são capazes de explicar toda a variação das estratégias de coping utilizadas pelos indivíduos (Antoniazzi et al., 1998). Somerfield e McRae (2000) explicam que, na última década, foi claramente demonstrado que os comportamentos de coping são muito influenciados pelas características do indivíduo, especialmente pelos traços de personalidade. As abordagens disposicionais aproximam-se da teoria do traço e focam nas estratégias de coping, que tendem a ser utilizadas pelas pessoas em situações estressantes variadas. São avaliadas como medidas de autorresposta, pois questionam o respondente acerca do modo habitual de agir perante situações estressantes. A abordagem situacional, em oposição, visa identificar como as estratégias de coping mudam perante diferentes situações.

Carver, Scheier, e Weintraub (1989) reconhecem que é "provavelmente óbvio" que existam estilos de coping, dado que as pessoas abordam cada contexto com um repertório de 
estratégias relativamente fixas através do tempo e das circunstâncias. A favor dessa perspectiva, defendem ainda que, provavelmente, há modos preferidos de coping em função das dimensões de personalidade. Esses autores referem-se a estilos de coping ou coping natural (disposition) para designar modos estáveis de enfrentamento que as pessoas utilizam nas situações estressantes que confrontam. Segundo essa perspectiva, os indivíduos possuem um conjunto de estratégias que se mantêm ao longo do tempo e situações, mais do tipo traço de personalidade. Holahan e Moos (1987) defendem que fatores sociodemográficos, como educação, nível socioeconómico, características de personalidade e fatores contextuais, influenciam o coping. De acordo com Suls et al. (1996), a Terceira Geração ainda se encontra em fase de evolução.

No contexto atual, podem-se observar duas grandes correntes conceituais de coping. A primeira linha aborda o coping segundo o ponto de vista disposicional, caracterizando-o como um traço ou estilo. Essa abordagem considera os diferentes estilos como disposições de personalidade, que transcendem a influência de contextos situacionais ou do tempo (Carver et al., 1989; Endler \& Packer, 1990; Holahan, Moos, \& Schaefer, 1996). Já a segunda corrente, denominada situacional, caracteriza o coping como um processo cognitivo, ativo e consciente de avaliação e resposta a situações de estresse. Essa abordagem pressupõe que as reações constituem um conjunto de pensamentos e comportamentos que ocorrem em resposta a eventos problemáticos específicos, sujeitos a se modificar tanto em função das particularidades da situação quanto através do tempo (Lazarus \& Folkman, 1984; Folkman et al., 1986).

Comumente, as duas abordagens são tratadas como representantes de teorias opostas, porém é importante dizer que elas não são mutuamente excludentes, e sim que interagem de forma frequente, explicando diferenças individuais em termos de coping e reação a situações de estresse (Carver et al., 1989; Holahan et al., 1996). Em geral, os estilos de coping têm sido mais relacionados às características de personalidade, enquanto as estratégias referem-se a ações cognitivas ou comportamentais adotadas no curso de um episódio particular de estresse. Aparentemente, os estilos de coping podem influenciar a extensão das estratégias selecionadas, embora costumem ser concebidos como fenômenos distintos e com diferentes origens teóricas (Ryan-Wenger, 1992). De qualquer forma, independentemente das divergências entre as abordagens citadas, nota-se que os modelos teóricos de estresse e coping possuem funções importantes para a investigação e que essas bases teóricas ajudam a focar nas questões de pesquisa, colocando-as em uma ordem lógica no sentido de ajudar a fornecer um quadro teórico, no qual os resultados possam ser estudados e interpretados (Folkman, 2009).

\section{O Modelo de Coping de Folkman e Lazarus}

Folkman e Lazarus postulam que a teoria do estresse e coping é uma estrutura para estudar o estresse psicológico. O modelo sustenta que o estresse é contextual, o que significa que se trata de um processo de relação entre a pessoa e o ambiente e que se transforma ao longo do tempo. Desse modo, ele é definido como uma situação avaliada pelo indivíduo como significativa e com demandas que excedem seus recursos para lidar com o respectivo evento (Folkman \& Lazarus, 1980; Folkman, 2010). Portanto o coping é conceituado como 
um conjunto de estratégias utilizadas pelas pessoas para adaptar-se a circunstâncias adversas ou estressantes (Antoniazzi et al., 1998), ou, em outras palavras, como todos os esforços de controle empreendidos em resposta a uma situação de estresse. É uma resposta com o objetivo de aumentar, criar ou manter a percepção de controle pessoal frente ao fator estressante (Savóia, Santana \& Mejias, 1996). Refere-se aos esforços cognitivos e comportamentais, ou seja, aos pensamentos e comportamentos que as pessoas usam para gerenciar, dominar, reduzir ou tolerar as demandas internas e externas criadas diante de uma situação estressora (Folkman \& Lazarus, 1980; Folkman, 2010).

Lazarus e Folkman (1984) propõem ainda que as respostas ao estresse podem ser de formas acomodativas ou manipulativas. Na resposta manipulativa, o indivíduo tenta alterar sua relação com o acontecimento estressante, o que poderia consistir em modificar o contexto, afastar-se ou, de qualquer outro modo, deixar de estar na presença do estressor; ou ainda procurar informações acerca da situação de tal forma que a possa compreender e prever acontecimentos relacionados a ela. Já na resposta acomodativa, ocorre uma inibição da ação diante do evento, impedindo o indivíduo de superar a adversidade ao acomodar-se à situação estressante através de sua reavaliação, alterando o ambiente interno por meio de medicamentos, álcool, relaxamento, mecanismos de defesa, meditação etc.

Na interação com o ambiente, inicialmente o indivíduo faz uma avaliação da situação estressora de forma cognitiva. Tal avalição diz respeito ao processo cognitivo por meio do qual um evento é avaliado em relação ao que se está em jogo (avaliação primária) e que recursos e opções de enfrentamento estão disponíveis para lidar com o fator de estresse (avaliação secundária) (Folkman \& Lazarus, 1980; Lazarus \& Folkman, 1984). A avaliação primária é o termo utilizado para definir a avaliação do significado atribuído pelas pessoas diante de uma situação de estresse. Ela é moldada por crenças pessoais, valores e objetivos. Já a avaliação secundária refere-se à avaliação que a pessoa faz de suas opções de enfrentamento. Essas opções são determinadas tanto pela situação, por exemplo, se há oportunidades de controlar o resultado, quanto pelos recursos físicos, psicológicos, materiais e espirituais de enfrentamento do indivíduo. As duas formas de avaliação determinam se a situação é avaliada como um dano ou uma perda, uma ameaça ou um desafio (Folkman, 2010). Logo, para selecionar as respostas que serão utilizadas para lidar com o evento estressor, a pessoa realiza a avaliação, a primária e a secundária. Na avaliação primária, é analisado se a circunstância é potencialmente prejudicial e ameaçadora, revelando quão importante esse evento é para o seu bem-estar. Se for compreendido como algo nocivo, o indivíduo inicia a avaliação secundária, na qual examina os recursos disponíveis para lidar com o respectivo evento (Snyder \& Dinoff, 1999).

Caso o acontecimento seja considerado um evento estressante, inicia-se então um processo cognitivo de avaliação do agente estressor. Nesse caso, existem três tipos principais de avaliações realizadas: 1. Perda ou danos, que se refere ao dano que já ocorreu; 2. Ameaça, que se refere ao dano ou perda que ainda não ocorreu, mas é antecipado; e 3. Desafio, que se refere a uma oportunidade antecipada de domínio ou ganho. Sendo assim, o indivíduo pode avaliar uma situação estressora como causadora de dano, se esta for contra seus valores pessoais ou o impedir de atingir seus objetivos, resultando em prejuízo psicológico. Já a ameaça é uma situação que antecipa o dano, ou seja, que ainda não ocorreu, mas é previsível. Contudo, ao contrário do dano, a ameaça permite organizar-se e preparar-se para 
o futuro. Nas situações de desafio, apesar de também serem estressantes, o indivíduo sente-se capaz de superá-las utilizando suas estratégias de enfrentamento de maneira eficaz. A ameaça e o desafio têm em comum a mobilização de estratégias de enfrentamento, mas, enquanto na ameaça o indivíduo foca no potencial lesivo da situação, gerando emoções negativas como ansiedade e medo, no desafio ele tenta pensar em estratégias para vencer o acontecimento, sendo acompanhado de sentimentos positivos. O grau em que uma pessoa experimenta o estresse psicológico, ou seja, o quanto se sente prejudicada, ameaçada ou desafiada, é determinado pela relação entre ela e o ambiente, uma vez que é definido tanto pela avaliação do que está em jogo quanto pela avaliação dos recursos de enfrentamento disponíveis (Folkman \& Lazarus, 1980).

Devido à sua característica dinâmica, o processo de coping pode ser eficaz em uma determinada situação, mas não em outra, dependendo, por exemplo, em que medida a situação é ou não controlável. Dessa forma, o que poderia ser considerado um enfrentamento efetivo no início pode ser considerado ineficiente mais tarde ou diante de outro evento estressante (Folkman \& Moskowitz, 2004). Lazarus e Folkman ressaltam também que os acontecimentos no decorrer da vida podem ser considerados benignos ou irrelevantes, não sendo necessária, nesses casos, uma resposta adaptativa especial.

Diante disso, o modelo de Folkman e Lazarus busca compreender o coping do ponto de vista das respostas cognitivas e comportamentais que as pessoas usam para gerenciar a angústia e resolver os problemas da vida diária que causam desconforto. A teoria coloca grande ênfase no papel da avaliação cognitiva na formação da qualidade da resposta emocional do indivíduo em uma relação pessoa-ambiente e as formas de lidar com o evento avaliado (Folkman \& Moskowitz, 2004). A teoria proposta envolve quatro conceitos principais: (1) Coping como um processo ou interação entre o indivíduo e o ambiente; (2) Com função de administrar a situação estressora, em vez de controlá-la ou dominá-la; (3) Processos de coping pressupondo a noção de avaliação, ou seja, como o fenômeno é percebido, interpretado e cognitivamente representado de forma mental pelo indivíduo; (4) Processo de coping constituído como uma mobilização de esforço, através da qual as pessoas irão empreender esforços cognitivos e comportamentais para administrar (reduzir, minimizar ou tolerar) as demandas internas ou externas que surgem da sua interação com o ambiente (Folkman \& Lazarus, 1980).

Originalmente, a teoria de estresse e coping de Folkman e Lazarus propõe um modelo que divide o fenômeno em duas categorias funcionais: coping focalizado no problema e coping focalizado na emoção (Figura 1). Essa construção baseou-se em análises fatoriais que geraram dois fatores principais utilizados pelos pesquisadores para definir os dois tipos de estratégias (Antoniazzi et al., 1998). O coping é visto nessa formulação como tendo duas funções principais: a regulação de emoções ou angústia (coping focalizado na emoção) e a gestão do problema que está causando o sofrimento (coping focado no problema). Ambas as formas são usadas em encontros mais estressantes, e as proporções relativas de cada tipo variam de acordo com a maneira como o encontro é avaliado (por exemplo, como tendo potencial de controle ou como não passível de controle) (Folkman \& Lazarus, 1980; Lazarus \& Folkman, 1984). 


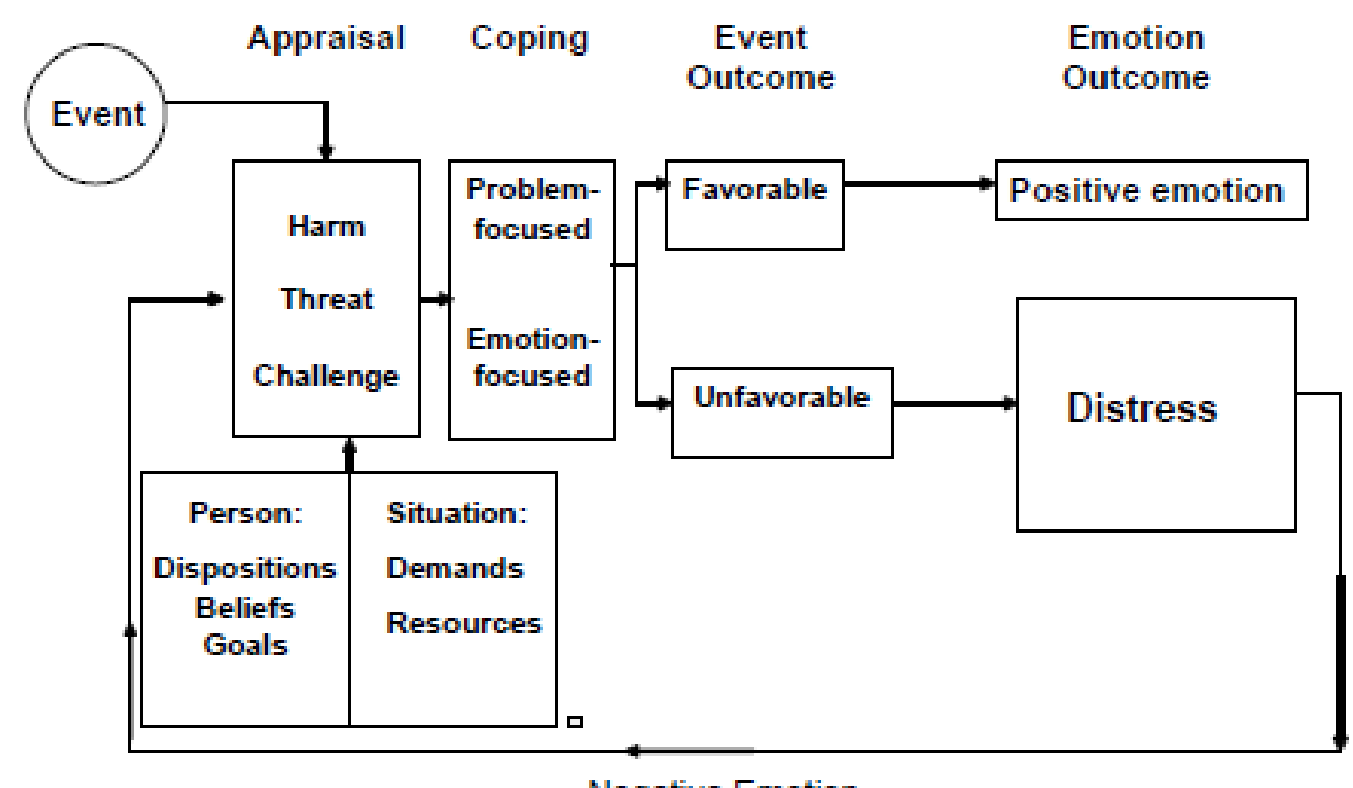

Negative Emotion

Figura 1. Modelo de Estresse e Coping Original (Lazarus \& Folkman, 1984).

O coping centrado no problema diz respeito às estratégias que são tomadas com referência no problema, ou seja, um plano de resolução dos eventos estressantes utilizando uma coleta de informações sobre a situação estressora para a tomada de decisão (Folkman, 2010). São consideradas estratégias de adaptação, uma vez que permitem a mudança da situação, seja pela tentativa de remover o problema, seja pela diminuição da capacidade de impacto da fonte estressora (Lazarus \& Folkman, 1984). No coping focado no problema, a pessoa iria engajar-se no manejo ou na alteração do problema ou situação causadora de estresse, visando controlar ou lidar com a ameaça, o dano ou o desafio. Em geral, trata-se de estratégias ativas de aproximação em relação ao estressor, como forma de planejamento e solução de problemas (Seidl, Tróccoli, \& Zannon, 2001). A função dessa estratégia é alterar o problema que está causando a tensão na relação entre a pessoa e o ambiente. A ação de coping pode ser direcionada interna ou externamente. Quando o coping focalizado no problema é dirigido para uma fonte externa de estresse, inclui estratégias tais como negociar para resolver um conflito interpessoal ou solicitar ajuda prática de outras pessoas. Quando dirigido internamente, costuma incluir reestruturação cognitiva, como a redefinição do elemento estressor (Antoniazzi et al., 1998).

O segundo estilo de coping é aquele em que a estratégia é focada na emoção e tem como característica o distanciamento, a fuga do problema e a busca por apoio emocional (Folkman, 2010). Descreve a tentativa de substituir ou regular o impacto emocional do estresse, resultado principalmente de processos defensivos que faz com que a pessoa evite confrontar, de forma realista, a ameaça estressora (Lazarus \& Folkman, 1984). O coping focalizado na emoção tem como função principal a regulação da resposta emocional causada pelo problema/estressor com o qual o indivíduo se defronta, podendo representar atitudes de afastamento ou paliativas em relação à fonte de estresse, como negação ou esquiva (Seidl, Tróccoli, \& Zannon, 2001). Esses esforços de coping são dirigidos a um nível somático e/ou de sentimentos, tendo por objetivo alterar o estado emocional. Fumar um cigarro, 
tomar um tranquilizante, assistir a uma comédia na TV e sair para correr são exemplos de estratégias dirigidas a um nível somático de tensão emocional. Sua função é reduzir a sensação física desagradável de um estado de estresse (Antoniazzi et al., 1998).

$\mathrm{Na}$ atualidade, um terceiro tipo de estratégia de coping vem sendo mencionado na literatura. Trata-se do coping focado, que se baseia em valores e crenças mais profundas e permite a revisão dos objetivos existenciais e a reordenação de prioridades por meio da experiência de vida, com o propósito de motivar e sustentar o enfrentamento e o bem-estar durante o tempo de dificuldades (Folkman, 2008, 2010). Este estilo de coping (Figura 2) regula as emoções positivas que desempenham funções importantes no processo de recuperação dos recursos para o enfrentamento do estresse, o que ajuda a transformar as avaliações de ameaças em avaliações de desafio e levar a esforços de motivação e de sustentação de enfrentamento a longo prazo. As estratégias de coping focado diferem qualitativamente das estratégias focadas na emoção, como o distanciamento, que regulam as emoções negativas. O coping focado se baseia em valores e crenças mais profundas na forma de estratégias como a revisão do objetivo, com ênfase nos pontos fortes ganhos com a experiência de vida e na reordenação de prioridades (Folkman, 2010).

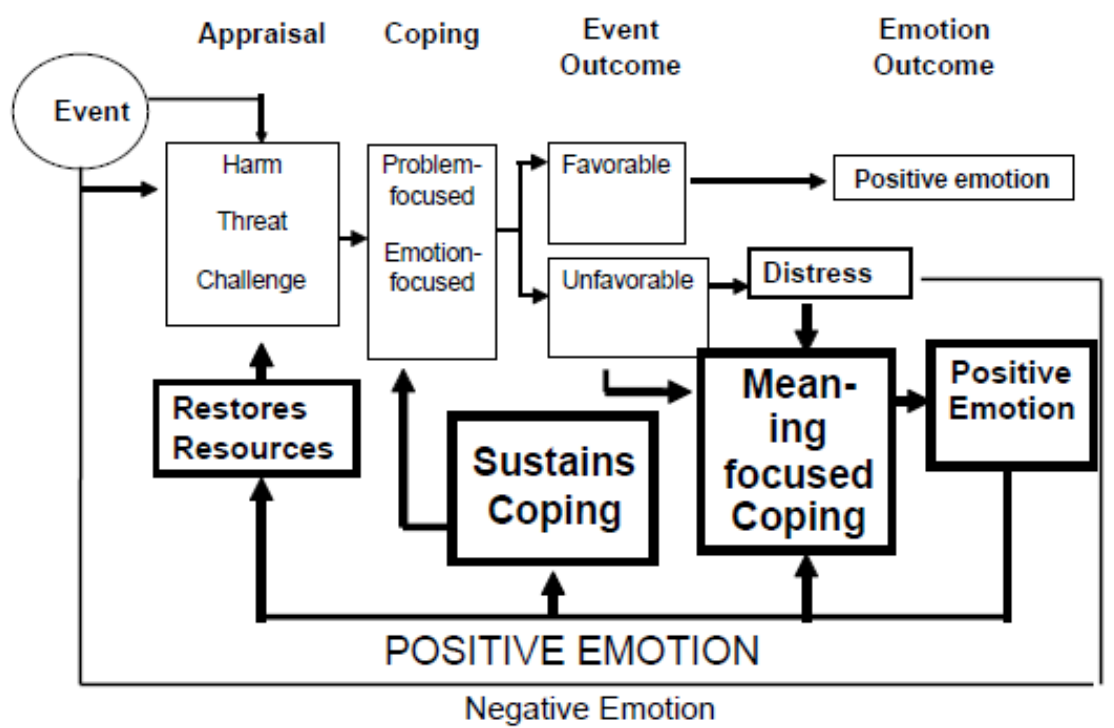

Figura 2. Modelo de Estresse e Coping Revisado (Folkman, 2008).

Vale destacar que esses estilos de coping não são necessariamente excludentes, pois sabemos que diferentes estratégias podem ser utilizadas de modo simultâneo para lidar com determinada situação estressora (Seidl et al., 2001). Os vários tipos de enfrentamento muitas vezes trabalham em conjunto, de forma que a regulação da ansiedade (coping focado na emoção) irá permitir que a pessoa se concentre em tomar uma decisão (coping focado no problema). Isto, por sua vez, é informado por meio de uma revisão dos valores subjacentes e os objetivos (coping focalizado). Idealmente, haveria independência entre esses processos, de modo a possibilitar uma previsão. Mas na realidade estamos diante de um sistema dinâmico de processos que são altamente interativos (Folkman, 2010). 


\section{Considerações Finais}

O modelo transacional do coping de Folkman e Lazarus é um dos mais utilizados pelos pesquisadores na atualidade. Nele, o coping é defendido como um processo ativo que resulta da avaliação que o indivíduo faz da relação entre si e o ambiente. Esses pressupostos implicam que as estratégias sejam ações deliberadas que podem ser aprendidas, usadas e descartadas (Antoniazzi et al., 1998). O respectivo modelo fornece uma estrutura útil para entender as formas como as ameaças à saúde, o enfrentamento e os comportamentos de saúde estão relacionados. Enfatiza a natureza transacional ou interacional do processo de enfrentamento e sugere que, quando as pessoas estão expostas a fatores de estresse, é desencadeado um processo de pensamento avaliativo, no qual o indivíduo passa a avaliar se o fator estressor é ou não uma ameaça ao seu bem-estar. Após essa fase, são feitas as escolhas das opções para enfrentar o estresse percebido, resultando, dessa forma, no comportamento de enfrentamento, na tentativa de controlar o evento estressor (Lazarus et al., 1985). Enfim, podemos sublinhar que as estratégias de coping não dizem respeito somente a uma exposição de conceito quanto à variabilidade na resposta a um evento estressor, mas sim em um importante caminho para novas intervenções no aspecto cognitivo e comportamental do indivíduo (Folkman \& Moskowitz, 2004).

Por fim, como limitação deste estudo, embora isso não invalide sua importância, cita-se o fato de esta ser uma revisão narrativa, ou seja, de cunho qualitativo, o que, de certa forma, não permite a reprodução de dados e o fornecimento de respostas "quantitativas".

\section{Referências}

Antoniazzi, A., Dell'Aglio, D., \& Bandeira, D. (1998). O conceito de coping: uma revisão teórica. Estudos de Psicologia, 3(2), 273-294. doi: 10.1590/S1413-294X1998000200006

Bernado, W. M., Nobre, M. R. C., \& Jatene, F. B. (2004). A prática clinica baseada em evidências. Buscando as evidências em fontes de informação. Revista da Associação Médica Brasileira, 50(1), 104-108. doi: 10.1590/S0104-42302004000100045

Brantley, P. J., \& Garret, V. D. (1993). Psychobiological approaches to health and disease. In P. Sutiker \& H. Adams (Eds.), Comprehensive handbook of psychopathology. New York: Plenum.

Brantley, P. J., \& Jones, G. N. (1993). Daily stress and stress-related disorders. Annals of Behavioral Medicine, 15(1), 17-25.

Carver, C., Scheier, M., \& Weintraub, J. (1989). Assessing coping strategies: A theoretically based approach. Journal of Personality and Social Psychology, 56(2), 267-283. doi: 10.1037/0022-3514.56.2.267

Chaves, E. C., Cade, N. V., Montovani, M. F., Leite, R. C. B., \& Spire, W. C. (2000). Coping: significados, interferência no processo saúde-doença e relevância para a enfermagem. Revista da Escola de Enfermagem da Universidade de São Paulo, 34(4), 370-375. doi: 10.1590/S0080-62342000000400008

Endler, N. S., \& Parker, J. D. A. (1990). Multidimensional assessment of coping: A critical evaluation. Journal of Personality and Social Psychology, 58(5), 844-854. doi: 10.1037/0022-3514.58.5.844 
Folkman, S., \& Lazarus, R. S. (1980). An analysis of coping in a middle-aged community sample. Journal of Health and Social Behavior, 21(3), 219-239. Disponivel em https:// www.jstor.org/stable/2136617

Folkman, S., \& Lazarus, R. (1985). If it changes must be a process: A study of emotion and coping during three stages of a college examination. Journal of Personality and Social Psychology, 48(1), 150-170. doi: 10.1037/0022-3514.48.1.150

Folkman, S., Lazarus, R. L., Dunkel-Schetter, C., DeLongis, A., \& Gruen, R. (1986). Dynamics of a stressful encounter: Cognitive appraisal, coping, and encounter outcomes. Journal of Personality and Social Psychology, 50(5), 992-1003. doi: 10.1037/0022-3514.50.5.992

Folkman, S., \& Moskowitz, J. T. (2004). Coping: Pitfalls and promise. Annual Review of Psychology, 55, 745-74. doi: 10.1146/annurev.psych.55.090902.141456

Folkman, S. (2008). The case for positive emotions in the stress process. Anxiety, Stress, \& Coping, January, 21(1), 3-14. doi: 10.1080/10615800701740457

Folkman, S. (2009). Commentary on the special section "Theory Based Approaches to Stress and Coping": Questions, answers, issues, and next steps in stress and coping research. European Psychologist, 14, 72-77. doi: 10.1027/1016-9040.14.1.72

Folkman, S. (2010). Stress, coping, and hope. Psycho-Oncology, 19, 901-908. doi: 10.1002/ pon.1836

Freud, A. (1937). The ego and the mechanisms of defence. London: Pub. by L. and Virginia Woolf at the Hogarth Press, and the Institute of psycho-analysis.

Freud, S. (1964). The neuro-psychoses of defense. In J. Strachey (Ed. \& Trans.), The standard edition of the complete psychological works of Sigmund. London: Hogarth. (Original work published 1894).

Haan, N. (1963). Proposed model of ego functioning: Coping and defense mechanisms in relationship to IQ change. Psychological Monographs, 77(8), 1-23. doi: 10.1037/h0093848

Haan, N. (1977). Coping and defending: Processes of self-environment organization. New York: Academic Press.

Holahan, C., \& Moos, R. (1987) Personal and contextual determinants of coping strategies. Journal of Personality and Social Psychology, 52(5), 946-955

Holahan, C. J., Moos, R. H., \& Schaefer, J. A. (1996). Coping, stress, resistance, and growth: conceptualising adaptive functioning. In M. Zeidner, \& N. S. Endler (Eds.), Handbook of coping: Theory, research, applications (pp. 24-43). New York: John Wiley \& Sons.

Holroyd, K. A., \& Lazarus, R. S. (1982). Stress, coping and somatic adaptation. In L. Goldberger, \& S. Breznitz (Eds.), Handbook of stress: Theoretical and clinical aspects (pp. 21-35). New York: Free Press.

Lazarus, R. S. (1966). Psychological stress and the coping process. New York: McGraw-Hill.

Lazarus, R. (1993). From psychological stress to emotions: a history of changing outlooks. Annual Review of Psychology, 44, 1-21. doi: 10.1146/annurev.ps.44.020193.000245

Lazarus, R. S., Delongis, A., Folkman, S., \& Gruen, R. (1985). Stress and adaptational outcomes: The problem of confounded measures. American Psychologist, 40(7), 770-779. doi: 10.1037/0003-066X.40.7.770

Lazarus, R., \& Folkman, S. (1984). Stress appraisal and coping. New York: Springer. Pais Ribeiro, J. L. (2005). Introdução à psicologia da saúde. Coimbra: Quarteto. 
Parker, J., \& Endler, N. (1992). Coping with coping assessment: A critical review. European Journal of Personality, 6, 321-344. doi: 10.1002/per.2410060502

Rother, E. D. (2007). Revisão sistemática x revisão narrativa. Acta Paulista Enfermagem, 20(2), 5-6. doi: 10.1590/S0103-21002007000200001

Ryan-Wenger, N. (1992). A taxonomy of children's coping strategies: A step toward theory development. American Journal of Orthopsychiatry, 62,256-263. doi: 10.1037/h0079328 Savóia, M. G., Santana, P. R., \& Mejias, N. P. (1996). Adaptação do inventário de estratégias de coping de Folkman e Lazarus para o português. Psicologia USP, 7(1-2), 183-201. doi: 10.1590/S1678-51771996000100009

Seidl, E. M. F., Tróccoli, B. T., \& Zannon, C. M. L. C. (2001). Análise fatorial de uma medida de estratégias de enfrentamento. Psicologia: Teoria e Pesquisa, 17(3), 225-234. doi: 10.1590/ S0102-37722001000300004

Seiffge-Krenke, I. (1993), Close friendship and imaginary companions in adolescence. New Directions for Child and Adolescent Development, 60, 73-87. doi: 10.1002/cd.23219936007

Snyder, C., \& Dinoff, B. (1999). Coping: Where have you been? In C. R. Snyder (Ed.), Coping: The Psychology of What Works (pp. 3-19). Oxford: Oxford University Press.

Somerfield, M., \& McCrae, R. (2000). Stress and coping research: methodological Challenges, theoretical advances, and clinical applications. American Psychologist, 55(6), 620-625.

Suls, J., David, J., \& Harvey, J. (1996). Personality and coping: Three generations of research. Journal of Personality, 64(4), 711-735. doi: 10.1111/j.1467-6494.1996.tb00942.x

Vaillant, G. (1977). Adaptation to life. Boston: Little, Brown.

Vaillant, G. (1994). Ego mechanisms of defense and personality psychopathology. Journal of abnormal psychology, 103, 44-50. doi: 10.1037/0021-843X.103.1.44

Recebido em: 26/10/2017

Última revisão: 11/03/2019

Aceite Final: 11/03/2019

\section{Sobre os autores:}

Ewerton Naves Dias - PhD. em Psicologia pela Faculdade de Psicologia e de Ciências da Educação da Universidade do Porto, Portugal. Mestre em Ciências da Saúde pela Universidade de São Paulo, Brasil. Especialista em Saúde Coletiva pela Universidade de São Paulo, Brasil. Docente da Universidade de Mogi das Cruzes, Brasil. E-mail: ewertonnaves@gmail.com, Orcid: http://orcid.org/0000-0002-5885-9331

José Luís Pais-Ribeiro - PhD. em Psicologia pela Universidade do Porto, Portugal. Docente da Faculdade de Psicologia e de Ciências da Educação da Universidade do Porto. E-mail: jlp@fpce.up.pt, Orcid: http://orcid.org/0000-0003-2882-8056 